\title{
La resolución de problemas como estrategia de desarrollo profesional docente: tensiones que se generan en el profesor
}

Problem solving as a teacher professional development strategy: tensions affecting teachers

\author{
Josefa Perdomo-Díaz ${ }^{1}$ \\ jperdomd@ull.edu.es \\ Universidad de La Laguna \\ Cristóbal Rojas \\ crojasb@dim.uchile.cl \\ Universidad de Chile \\ Patricio Luis Felmer \\ pfelmer@dim.uchile.cl \\ Universidad de Chile
}

\section{Resumen:}

Los programas de desarrollo profesional docente tienen como objetivo generar cambios en los profesores, en sus conocimientos, sus creencias o sus prácticas. Para que esos cambios se produzcan, la experiencia vivida en el programa de desarrollo profesional debe provocar que el profesor se cuestione aspectos relacionados con su profesión. En este artículo presentamos una estrategia de desarrollo profesional para profesores de matemática de todos los niveles educativos, centrada en el uso de la resolución de problemas, y mostramos un análisis de las tensiones que este tipo de estrategia provoca en los profesores participantes. Para ello se realiza el estudio de un caso, correspondiente a un taller denominado RPAula, realizado

\begin{abstract}
:
Teacher professional development programs aim to generate changes in teachers, in their knowledge, beliefs or practices. For these changes to occur, the professional development experience should cause the teacher to question aspects related to his/her profession. In this article, we present a problem-solvingbased professional development strategy for mathematics teachers and we offer an analysis of the tensions which emerged. To do this, we carried out a case study based on a workshop called RPAula that took place in Chile with elementary teachers. The results show that participation in this workshop causes tensions related to teachers' expectations about their students, their beliefs about teaching and
\end{abstract}

1 Dirección para correspondencia (correspondence address):

Josefa Perdomo-Díaz. Departamento de Análisis Matemático, Avda. Astrofísico Francisco Sánchez, s/n. 38206. La Laguna. Santa Cruz de Tenerife. Tenerife (España). 
La resolución de problemas como estrategia de desarrollo profesional docente:

tensiones que se generan en el profesor

Cristóbal Rojas, Josefa Perdomo-Díaz y Patricio luis Felmer

en Chile, con profesores de educación básica. Los resultados muestran que la participación en este taller provoca tensiones relacionadas con las expectativas que los profesores tienen hacia sus estudiantes, sus creencias acerca de la enseñanza y el aprendizaje y sus modelos de enseñanza de la matemática.

\section{Palabras clave:}

Formación docente; desarrollo profesional; resolución de problemas; matemáticas; tensiones. learning, and their models of mathematics teaching.

\section{Key words:}

Teacher training, professional development, problem solving, mathematics, tensions.

\section{Résumé:}

Les programmes de développement professionnel des enseignants visent à générer des changements chez les enseignants, dans leurs connaissances, leurs croyances ou leurs pratiques. Pour que ces changements se produisent, l'expérience du programme de perfectionnement professionnel doit amener l'enseignant à remettre en question des aspects liés à sa profession. Dans cet article, nous présentons une stratégie de développement professionnel pour les enseignants de mathématiques de tous les niveaux d'éducation, centrée sur l'utilisation de la résolution de problèmes. Nous montrons une analyse des tensions que ce type de stratégie provoque chez les enseignants participants. Pour ce faire, une étude de cas correspondant à un atelier appelé RPAula, réalisé au Chili, avec des enseignants de l'école primaire est réalisée. Les résultats montrent que la participation à cette formation provoque des tensions liées : aux attentes des enseignants vis-à-vis de leurs élèves ; à leurs croyances en matière d'enseignement et d'apprentissage ; et à leurs modèles d'enseignement des mathématiques.

\section{Mots clés:}

Formation des enseignants; perfectionnement professionnel; résolution de problèmes; mathématiques; tension.

Fecha de recepción: 9-5-2018

Fecha de aceptación: 13-9-2018

\section{Introducción}

Los programas de desarrollo profesional docente son parte esencial de cualquier sistema educativo; de ellos depende, en gran medida, que las reformas educativas Ileguen a hacerse efectivas en el aula. Las propuestas de desarrollo profesional de calidad buscan ayudar a que los docentes profundicen en sus conocimientos sobre la disciplina y/o sobre los procesos de pensamiento y aprendizaje de los estudiantes, y modifiquen sus prácticas (Borko, 2004). Este proceso de cambio en el profesor es complejo ya que requiere que se involucre cognitiva y emocionalmente, 
que esté dispuesto a examinar sus propios conocimientos, creencias y prácticas y esté receptivo a propuestas alternativas (Ávalos, 2011).

Según Guskey (2002), una de las razones que hay detrás del fracaso de algunas propuestas de desarrollo profesional docente es que no se presta suficiente atención al proceso por el que se produce el cambio en el profesor. En este sentido, Ávalos (2011) señala que "si hay una brecha entre las creencias y las prácticas sugeridas, el cambio solo ocurrirá si se reconoce esa brecha" (p. 15). Esas diferencias entre lo que se presenta, o lo que se percibe, en un programa de desarrollo profesional y los conocimientos, creencias o experiencias del profesor generan tensiones en el docente. Analizar esas tensiones aporta información relevante para el desarrollo y rediseño del propio programa de formación docente, para la formulación de otras propuestas y para profundizar en la comprensión del proceso de cambio del profesor.

Las investigaciones sobre las tensiones que genera la participación en programas de desarrollo profesional (PDP) en los docentes son aún muy escasas. Tan, Chang, y Teng (2015) analizan los dilemas y las tensiones que les surgen a los profesores cuando tienen que decidir si participar o no en un PDP. Los autores señalan que las creencias de los docentes y la expectativa social sobre ellos tienen implicaciones directas en esta decisión, e identifican tensiones entre las necesidades personales del docente versus las de su organización, la responsabilidad de actualizarse versus estar con sus estudiantes y el desarrollo profesional obligatorio versus el voluntario, según sus propias necesidades. Nipper et al. (2011) identifican tensiones que aparecen cuando las expectativas de los docentes no coinciden con su experiencia en el PDP, distinguiendo entre tensiones pedagógicas y tensiones matemáticas. Las tensiones pedagógicas son aquellas que surgen a raíz de la relación que se da en el PDP entre el monitor y los participantes, por ejemplo, en relación con la factibilidad de usar en el aula la metodología observada en el PDP. Las tensiones matemáticas se dan en la relación del docente con el contenido matemático del programa, que en el caso de esta investigación era geometría.

En este artículo se presenta el análisis de las tensiones manifestadas por los docentes, al finalizar la primera sesión de un taller de desarroIlo profesional para profesores de matemática de Educación Básica, denominado Taller RPAula. El objetivo general de este taller es ofrecer a los participantes la oportunidad de conocer un modelo de trabajo de 
La resolución de problemas como estrategia de desarrollo profesional docente: tensiones que se generan en el profesor

Cristóbal Rojas, Josefa Perdomo-Díaz y Patricio luis Felmer

la resolución de problemas matemáticos en el aula, implementarlo y reflexionar sobre su puesta en práctica. El enfoque de resolución de problemas sobre el que se sustenta el diseño del taller es aquel que considera que un problema es cualquier actividad matemática que suponga un desafío para la persona que lo enfrenta, de manera que le genere un interés por resolverlo, no conozca un procedimiento directo que le lleve a la solución pero sí se sienta capaz de abordarlo, sin importar el contexto en el que esté planteado (Perdomo-Díaz \& Felmer, 2017). La resolución de problemas es considerada, en este taller, como una habilidad que se desarrolla en la medida en que se practica, un proceso con diferentes acercamientos, en el que cada individuo pone en juego sus conocimientos, tratando de conectarlos e integrarlos en la búsqueda de una solución a la situación planteada. Esta perspectiva de la resolución de problemas tiene una amplia tradición en Educación Matemática que se mantiene vigente (Liljedahl, Santos-Trigo, Malaspina y Bruder, 2016; Felmer, Pehkonen \& Kilpatrick, 2016).

El diseño general del Taller RPAula busca movilizar la concepción que los participantes tienen de sí mismos como resolutores de problemas, además de su sistema de creencias acerca de la matemática, su enseñanza y las expectativas que tienen de sus estudiantes respecto del aprendizaje de la matemática. Para ello se utiliza un enfoque centrado en hacer y reflexionar, con espacios donde los profesores resuelven problemas en un escenario donde la relación monitor-docente-matemática modela la relación docente-estudiante-matemática; seguidamente reflexionan sobre los elementos principales del modelo, planifican actividades de resolución de problemas con sus alumnos, las implementan y analizan. Esto se hace en 8 sesiones, durante un curso escolar (PerdomoDíaz \& Felmer, 2017).

En la primera sesión del Taller RPAula, el hacer consiste en situar al docente en el papel de resolutor de problemas, de forma que la experiencia vivida sirva como modelo para el trabajo de la resolución de problemas en su aula. La sesión finaliza con una discusión plenaria, en la que los docentes reflexionan sobre cómo se sintieron, qué dificultades encontraron, qué cosas les Ilamaron la atención. El objetivo de esta discusión es comenzar a hacer explícitas las características principales de la propuesta de trabajo en resolución de problemas: el tipo de actividad que se utiliza, la distribución de los participantes y la interacción que se produce entre los ellos y con el monitor, modelo de interacción entre 
los docentes y sus estudiantes. Es en esta discusión final donde se espera que afloren las primeras tensiones en los docentes, al comparar la experiencia vivida en el taller con la forma en que ellos trabajan la resolución de problemas con sus estudiantes.

Surge así la pregunta que guía esta investigación: ¿qué tipo de tensiones declaran los participantes del Taller RPAula, en relación con el modelo de trabajo en resolución de problemas que experimentan en la primera sesión del taller?

En este artículo se presenta un estudio exploratorio de dichas tensiones, a partir del análisis de un caso, correspondiente a un Taller RPAula, realizado en Santiago de Chile, en el que participaron 24 profesores de Educación Básica (de $1 .^{\circ}$ a $8 .^{\circ}$ ). Los datos recogidos y analizados corresponden a la grabación en video de la discusión plenaria realizada al finalizar la primera sesión del taller.

La investigación se realiza desde una perspectiva situada, en la cuál el aprendizaje se concibe como cambios que se producen participando en actividades sociales organizadas, de forma que tanto el contexto como las actividades con las que un individuo aprende constituyen una parte fundamental de lo que aprende (Greeno, Collins, \& Resnick, 1996; citado en Borko, 2004). Tal y como ocurría en el trabajo de Nipper et al. (2011), las tensiones pueden estar relacionadas con aspectos matemáticos o pedagógicos, y están fuertemente ligadas al diseño del taller, por lo que el marco de referencia para el análisis incluye, por una parte, las relaciones involucradas en el Taller RPAula, y por otra parte, distintas categorías de tensiones que otros estudios han identificado en los formadores de profesores y en los profesores, cuando imparten docencia.

\section{Desarrollo profesional docente}

El desarrollo profesional docente trata sobre el aprendizaje de los profesores, aprender cómo aprender y transformar su conocimiento en prácticas beneficiosas para sus estudiantes (Ávalos, 2011). Los PDP dirigidos a docentes son esfuerzos sistemáticos para provocar cambios en sus prácticas de aula, sus actitudes y creencias (Guskey, 2002).

Distintas investigaciones han proporcionado valiosa información sobre ciertas características que contribuyen a que un PDP dirigido a profesores sea efectivo, que tienen que ver con la duración, el contenido 
La resolución de problemas como estrategia de desarrollo profesional docente: tensiones que se generan en el profesor

Cristóbal Rojas, Josefa Perdomo-Díaz y Patricio luis Felmer

y la metodología (Garet, Porter, Desimone, Birman, \& Suk Yoon, 2001; Marrongelle, Sztajn, \& Smith, 2013). Dos de los aspectos que se mencionan es que incluyan un foco disciplinar y un foco en las prácticas de enseñanza. De esta forma, entre los objetivos del programa debe estar que el profesor participante se vea involucrado en actividades propias de una disciplina, como la resolución de problemas matemáticos, y que incorpore ideas del PDP en su enseñanza (Borko, 2004). El Taller RPAula incluye estas dos componentes en su diseño, dedicando un amplio espacio a actividades donde los participantes resuelven problemas y utilizando esas instancias como modelo para que los docentes planifiquen e implementen Actividades de Resolución de Problemas en el Aula (ARPAs) con sus estudiantes (Perdomo-Díaz \& Felmer, 2017).

Tal y como señalan Nipper et al. (2011), los profesores que participan en un PDP experimentan una diversidad de tensiones, fruto de las relaciones que se establecen dentro del propio programa y la proyección que los docentes hacen de su aplicación en el aula. Resulta útil por tanto, establecer un modelo de relaciones asociado al diseño y desarrollo del Taller RPAula, como punto de partida para el análisis de las tensiones que declaran los docentes después de haber participado en la primera sesión, con foco disciplinar.

Los PDP son instancias que pueden ser descritas en términos del triángulo didáctico (profesor-estudiante-contenido), considerando al monitor de la estrategia como el profesor y a los participantes como los estudiantes. En el caso de propuestas dirigidas al profesorado, un tema que preocupa a los participantes es su práctica habitual, es decir, cómo se relacionarán sus estudiantes con la metodología expuesta o con el material que se les brinda para trabajar en sus aulas. De manera natural, el triángulo instruccional se expande, dando lugar a un rombo instruccional (Nipper y Sztajn, 2008), donde el cuarto vértice representa a los estudiantes que se verían afectados por la participación de su profesor en el PDP.

En el Taller RPAula, el contenido es la resolución de problemas, bajo la perspectiva descrita en la introducción. Por otra parte, el diseño del taller incluye mostrar un modelo de enseñanza centrado en el estudiante, donde los problemas se resuelven en grupos formados al azar. Esto introduce un nuevo elemento en el modelo de relaciones del PDP, que es la relación del docente con sus compañeros. Por tanto, las relaciones que se establecen en el Taller RPAula, pueden ser representadas con el esque- 
ma de la Figura 1, el cuál servirá de base para estructurar el análisis de las tensiones que los docentes manifiestan durante la discusión plenaria con la que finaliza la primera sesión del taller.

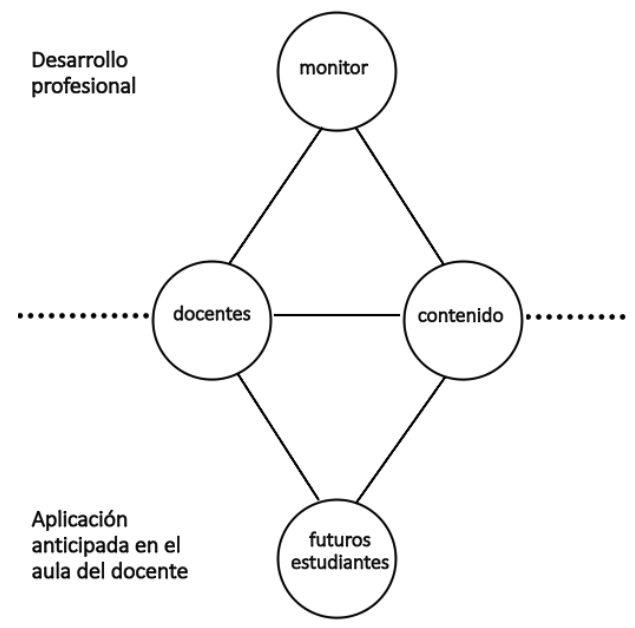

Figura 1. Modelo de relaciones involucradas en el Taller RPAula.

\section{Tensiones}

Para llevar a cabo este estudio, consideramos que una tensión es un sentimiento que se produce en una persona cuando se ve enfrentada a problemas con distintas soluciones (Lampert, 1985) o situaciones que pueden ser enfocadas de diversas maneras. Pueden estar provocadas por fuerzas o ideas que se contraponen o que presentan variedad de alternativas frente a un mismo escenario y que generan sentimientos de dudas internas, que pueden tener mayor o menor intensidad (Berry, 2008).

Berry (2008) hace notar una dificultad en la identificación de las tensiones y es su fuerte interdependencia; no vienen como paquetes separados y bien definidos, sino que se entrelazan entre sí. Por tanto, contar con modelos y clasificaciones iniciales, resulta muy útil para el análisis y estudio de las tensiones.

La literatura recoge dos corrientes principales en el estudio de las tensiones, una donde los sujetos analizados son profesores y otra donde lo son formadores de profesores. 
La resolución de problemas como estrategia de desarrollo profesional docente: tensiones que se generan en el profesor

Cristóbal Rojas, Josefa Perdomo-Díaz y Patricio luis Felmer

Uno de los primeros trabajos lo realiza Lampert (1985), observando la sala de clases desde la perspectiva de un docente. Esta autora considera que las tensiones nacen cuando los docentes se enfrentan a dilemas, entendidos como problemas con distintas soluciones. Lampert analiza las tensiones a las que se ve sometido un docente, en términos de las decisiones que debe ir tomando. Esta idea es retomada por Berry (2008), en el contexto de la formación inicial docente, para analizar los sentimientos de dudas internas que experimentan los formadores de futuros profesores. En este modelo se considera la deliberación a la que se ve sometido el individuo durante el proceso de toma de decisión, por sobre la decisión misma, identificándose ocho tipos de tensiones: Decir versus Crecer, Confianza versus Incertidumbre, Acción versus Intención, Seguridad versus Desafío, Planificar versus Ser sensible y Valorar versus Reconstruir la Experiencia.

El trabajo de Berry se ha convertido en referente de diversas investigaciones. Por ejemplo, Rouleau y Liljedahl (2017) parten de su clasificación para analizar las tensiones de un docente en su sala de clases. Estos autores consideran las tensiones como los conflictos que pueden surgir cuando los maestros de matemáticas encuentran una diferencia entre lo que quieren hacer y lo que se les pide que hagan, o entre lo que quieren hacer y lo que saben hacer. Los autores reportan tensiones ya identificadas por Berry (Decir versus Crecer, Confianza versus Incertidumbre y Acción versus Intención) y añaden algunas nuevas que tienen que ver con la relación de la profesora con los padres de sus alumnos, con sus colegas y con las pruebas estandarizadas.

En un artículo posterior, Rouleau (2017) vuelve a utilizar el modelo de Berry (2008) para estudiar las tensiones que experimentan los mentores matemáticos, es decir, aquellas personas que de alguna manera guían a los profesores, ayudándoles a mejorar su práctica en la enseñanza de la matemática. En este trabajo se encuentran tres de las tensiones del modelo de Berry (Seguridad versus Desafío, Valorar versus Reestructurar la Experiencia, Confianza versus Incertidumbre) y aparece una nueva, que denomina Iniciativa versus Barreras Sistémicas, que hace referencia a las dificultades que los mentores encuentran para trabajar con asistentes de la enseñanza.

En el contexto de los PDP docente, las tensiones pueden darse tanto en los docentes participantes como en la persona que imparte el programa de formación, el monitor o facilitador. Para examinar la mirada del 
monitor, Brown (2010) realiza un estudio de sí misma como monitora de un taller de desarrollo profesional docente y describe tensiones generadas en torno a dos situaciones: los objetivos personales compiten con los del programa y el dilema entre ser explícita respecto al tema de discusión o dejar que éste emane de los docentes. A este conjunto de tensiones, hay que añadir las ya mencionadas en la introducción, indicadas por Nipper et al. (2011) en relación con los participantes en un PDP, quienes manifestaban tensiones sobre aspectos pedagógicos y también sobre los contenidos matemáticos del programa.

El modelo de relaciones del Taller RPAula (Figura 1) y las clasificaciones de tensiones anteriores son dos herramientas esenciales para analizar las tensiones que declaran los docentes al finalizar la primera sesión del taller. Este análisis permitiría comprender mejor el proceso de cambio al que se ve sometido el profesor cuando participa en este PDP.

\section{Metodología}

\section{Participantes}

Se realiza el estudio de un caso, correspondiente a un Taller RPAula realizado en 2015, en Santiago de Chile. En este taller participaron 24 profesores de Educación Básica $\left(1^{\circ}\right.$ a $\left.8^{\circ}\right)$, todos provenientes de escuelas públicas (Tabla 1).

Tabla 1. Características de los docentes participantes del taller.

\begin{tabular}{lccc}
\hline Sexo & Mujer & Hombre & No indica \\
\cline { 2 - 4 } Formación como profesor & 14 & 4 & 6 \\
\cline { 2 - 4 } & Básica General & $\begin{array}{c}\text { Básica, con mención } \\
\text { en Matemática }\end{array}$ & Otro \\
\cline { 2 - 4 } & 9 & 8 & 7 \\
\hline
\end{tabular}

\section{Contexto: la primera sesión del Taller RPAula}

De las 8 sesiones del taller, la primera se dedica exclusivamente a la resolución de problemas por parte de los docentes; tiene como foco principal que los profesores participantes resuelvan problemas, con una dinámica de interacción caracterizada por el trabajo en grupo y la 
La resolución de problemas como estrategia de desarrollo profesional docente:

ausencia de respuestas por parte del monitor, quién interviene siempre formulando preguntas. Los problemas propuestos son actividades que incluyen una amplia variedad de contenidos todos ellos estudiados en la etapa de Educación Primaria (números, geometría, álgebra, etc.), pueden resolverse utilizando diversas estrategias y procedimientos y están planteados de forma que permitan simplificaciones y extensiones. Se comienza por uno que sea desafiante y a la vez abordable, buscando generar un clima de autoconfianza (Figura 2a); se va aumentando la complejidad de manera progresiva, alcanzando un nivel máximo más o menos a mitad de sesión (Figura 2b).

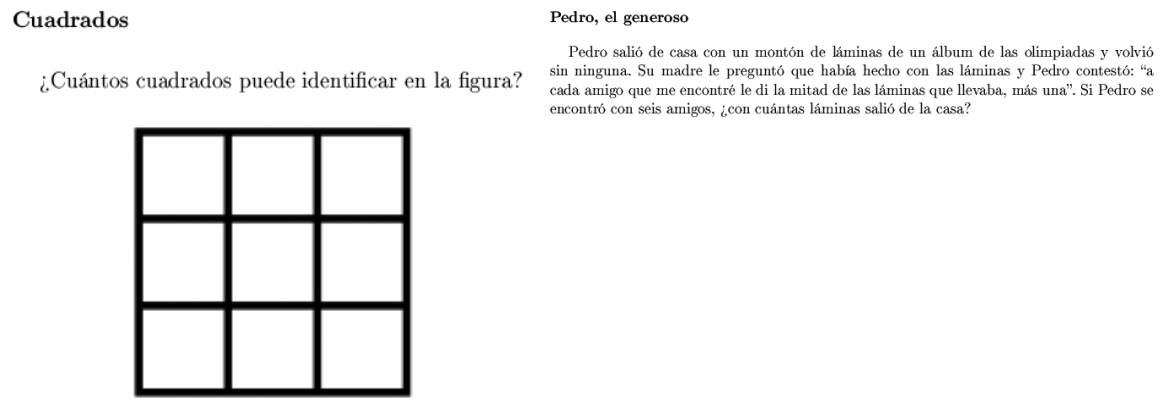

Figura 2. Problemas trabajados en la primera sesión del Taller RPAula.

La sesión se inicia conformando grupos al azar y entregando el primer problema a cada docente. Cada grupo trabaja a sus propio ritmo. Los participantes pueden Ilamar al monitor si tienen una duda o si consideran que el grupo ya ha resuelto el problema. El monitor responde interactuando solo mediante preguntas, tanto para resolver las dudas como para verificar que todos los integrantes del grupo han comprendido la(s) solución(es) que ellos mismos presentan. Esta sería la parte de la sesión dedicada a hacer.

La reflexión se da al final de la sesión, en formato de discusión plenaria. El objetivo de esta discusión plenaria es que los profesores reflexionen sobre cómo se han sentido durante la actividad de resolución de problemas y comiencen a identificar algunas características de la dinámica de trabajo como el tipo de actividad que se les propone, el trabajo grupal, la interacción entre el monitor y los docentes, las preguntas, la verificación de soluciones, simplificaciones y extensiones. Estos temas son abordados conforme el monitor va haciendo preguntas a los docen- 
tes. En este momento afloran y se hacen explícitas distintas tensiones que se producen en el profesor al comparar la experiencia que han vivido en el taller con su práctica habitual.

\section{Datos y procedimiento de análisis}

Los datos analizados corresponden a la grabación en vídeo de la discusión plenaria realizada en la primera sesión del Taller, con una duración aproximada de 40 minutos.

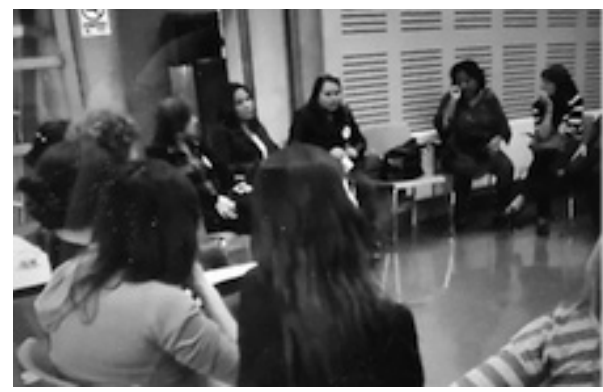

Figura 3. Imagen de la discusión plenaria del Taller RPAula.

Para realizar el análisis, en primer lugar se transcribió la plenaria, utilizando la etiqueta [M] para las intervenciones del monitor y [D] para las de los docentes. En el caso de que en un mismo episodio interviniera más de un profesor, se utilizaron las etiquetas [D1], [D2], etc. Posteriormente, se identificaron episodios de tensiones atendiendo a la definición dada en el marco conceptual, que fueron clasificados según la relación en la cual la tensión tiene lugar (Figura 1). Finalmente se identificaron los temas subyacentes a la tensión, utilizando como base distintas clasificaciones de tensiones presentes en la literatura (por ejemplo, Berry, 2008; Rouleau y Liljedahl, 2017) y generando nuevas categorías en caso necesario.

El análisis se realizó mediante un proceso iterativo, donde uno de los autores realizaba la transcripción y una primera identificación y clasificación de las tensiones, que era posteriormente contrastada por los otros dos autores. 
La resolución de problemas como estrategia de desarrollo profesional docente: tensiones que se generan en el profesor

Cristóbal Rojas, Josefa Perdomo-Díaz y Patricio luis Felmer

\section{Resultados}

En total se identificaron 10 episodios de tensiones, concentradas en las relaciones entre el docente y la resolución de problemas (2), el docente y sus estudiantes (6) y el docente y el sistema (2). Los participantes de este taller no manifestaron tensiones en la relación Docente-Monitor y tampoco en la relación Docente-Grupo.

\section{Tensiones en la relación Docente-Resolución de problemas}

En dos episodios, algunos de los participantes indicaron que, durante la resolución de los problemas, se habían sentido inseguros con respecto a sus conocimientos y habilidades matemáticas. Esto puede observarse en expresiones como "compartir la estrategia que uno tiene hace que uno se vaya dando cuenta de qué es lo que le falta a uno también por mejorar", "totalmente perdida, sobre todo, por ejemplo, en la tarde, que era con materias que no conocía", "tengo que aprender esto, tengo que repasar esto, tengo que practicar esto".

\section{Episodio 1.}

[M] Docente (refiriéndose con su nombre), ¿cómo se sintió?

[D] Bien. Lo que sí, en la mañana me sentí como mucho más [...] Mentalmente parece que más abierta con la solución de los problemas que ahora. Ahora me sentí como más, como más cerrada, me costó más encontrar laaa.. la.., laa...No sé si era porque era ya después de almuerzo, mi mente estaba ya con un poquito más de flojera. Pero bien; estuve [...] tuve buenos grupos de trabajo, entonces siempre el compartir la estrategia que uno tiene hace que uno se vaya dando cuenta de qué es lo que le falta a uno también por mejorar. Entonces eso también facilita todo para llegar a una buena, a un buen final.

\section{Episodio 2.}

[M] ¿Qué se siente cuando yo estoy muy perdido y tengo un compañero al lado que me ilumina el camino?

[D] Yo le puedo decir. Porque la verdad es que yo [...] no hago matemáticas $y$, la verdad de las cosas, es que totalmente perdida, sobre 
todo, por ejemplo, en la tarde, que era con materias que no conocía. Quizás se tiene que tener el conocimiento previo para [...] ¿te fijas?

[M] ¿Te refieres más como al de las fracciones? ya ajam

[D] Exactamente. Entonces, obviamente que bien porque las personas que están a tu lado te acompañan, te ayudan, te enseñan. Y tú dices bueno, yo tengo que aprender esto, tengo que repasar esto, tengo que practicar esto, pero regio porque la persona que está al lado tuyo te ayuda, te colabora.

Estas tensiones estarían en el grupo de las tensiones matemáticas descritas por Nipper et al. (2011), aunque difieren de las indicadas por estos autores. Nipper et al señalan que, cuando los docentes participan en un PDP, esperan encontrarse con contenidos con un nivel de dificultad similar al nivel de dificultad con que realizan sus clases. En muchas ocasiones, durante el taller encuentran que los contenidos son de un nivel superior, pues el taller está pensado para mejorar su conocimiento matemático, y ésto les genera tensiones. En el caso estudiado, los participantes del Taller RPAula no manifestaron ninguna tensión relacionada con el hecho de que el contenido fuera inadecuado para usarlo con sus estudiantes, pero sí mostraron tensiones sobre sus propios conocimientos y habilidades matemáticas y la necesidad de mejorarlos. Identificar esta brecha en sus propios conocimientos matemáticos es importante para el desarrollo profesional de los docentes (Ávalos, 2011; Borko, 2004).

\section{Tensiones en la relación Docente-Estudiantes}

Los siguientes seis episodios muestran reflexiones en torno a la relación entre el profesor y sus alumnos, fruto de la comparación que los docentes realizan, de manera natural, entre la dinámica de trabajo que han vivido en el taller y sus prácticas pedagógicas. Los tópicos de discusión giran en torno al modelo de presentación de la resolución de problemas que usan generalmente en el aula, el tratamiento de los errores de los estudiantes, el trabajo en grupo, la gestión del tiempo y el monitoreo del trabajo de los estudiantes.

Episodio 3. En este episodio se observa una tensión entre distintos modelos de gestión de la actividad de resolución de problemas: el utilizado 
La resolución de problemas como estrategia de desarrollo profesional docente: tensiones que se generan en el profesor

Cristóbal Rojas, Josefa Perdomo-Díaz y Patricio luis Felmer

por los profesores en su clase, que consiste en entregar una pauta de resolución, con un único método y una única solución, y el vivido en el taller, que promueve que sea el resolutor quién descubra una estrategia de resolución y favorece el uso de diferentes procesos de resolución.

[D1] Agregando a lo que dice ella de también buscar otra estrategia para contestar el mismo problema. Para el mismo resultado, porque uno por lo general se queda "ah, ya, ya me salió, ya tomemos el otro", pero en este caso también nos ayudaba a buscar a lo mejor otra forma que también nos ayudara a llegar al resultado. Y uno, basado en la experiencia, igual lo hace poco, uno le enseña la misma estrategia que tiene uno, no la que sea más cómoda para ellos. Porque nosotros buscábamos la más cómoda para nosotras, pero nosotras muchas veces le damos la estrategia, no los dejamos ahí descubriendo, como nos pasó a nosotros aquí.

[D2] Sabes que sí. Es verdad lo que dice ella. Yo me, me autoevalué. Me miré a mí misma haciendo clases [gesticula como escribiendo en una pizarra]. Todos los niños mirando sentados. "Ya, resolvamos el problema. Ya, acá, cual es el enunciado. Busquemos, ¿cuál es la palabra clave?, ¿qué proceso hacemos?" Todo bien estructurado, pero nunca he dejado que los niños busquen el camino de ellos.

Episodio 4. En este episodio la tensión involucra diferentes formas de actuar ante los errores de los estudiantes. Frente a la experiencia del docente, que reconoce optar por hacer notar explícitamente a los estudiantes que sus desarrollos están mal, aparecen formas alternativas para que el estudiante revise sus procesos de resolución y note sus errores como formular una pregunta que le haga revisar los datos del problema.

[D] ¿Y sabes lo que no ocupabas tú? la palabra que nosotros siempre ocupamos, o al menos yo. Es: “¡No!, está malo". Era como "no digo que está malo, [...] podrías pensarlo mejor" pero no el "jestá malo!" [M] Y en lugar de decir "¡No!, está malo" o "Te equivocaste" o lo que fuera ¿Qué hacía yo? ¿Cuál era mi rol?

[D] Claro, como: "a ver, ve otra opción", "no sé, lee bien, lee de nue$v o^{\prime \prime}$... Porque uno lo primero que le dice es ¡no, malo! pero si tú nos decías "no, sabes qué, lee de nuevo" entonces uno... "¡ah! Claro". Pero no era el "iNo!" que uno siempre le dice [...] el "¡No!". 
Los dos episodios anteriores guardan cierta relación con la categoría "Decir versus Crecer" descrita por Berry (2008), confrontando un modelo de enseñanza en el que el docente indica a los estudiantes cómo hacer las cosas, frente a un modelo en el que el docente ofrece oportunidades para que aprendan a hacerlo, sin indicar de manera exacta y precisa cómo lograrlo. Mientras trabajan en la resolución de un problema, los docentes generan preguntas con respecto al enunciado y escogen qué estrategia utilizar para resolverlo. El monitor del taller no intenta cambiar la estrategia escogida por los docentes y responde a las preguntas mediante otras preguntas, que tienen por función ayudar a los docentes a responder sus propias dudas, desarrollando conocimiento autónomo. Esta forma de manejar las dudas genera una tensión en los docentes, cuando la comparan con lo que ellos hacen habitualmente en sus aulas. En esta situación, "decir" corresponde con responder las preguntas directamente e indicar las estrategias a seguir y "crecer" a no responder directamente y permitir el uso de la estrategia elegida por el estudiante. Algo similar ocurre con el episodio 4; en el Taller RPAula, cuando los docentes exponen su solución, si el monitor encuentra un error, hace una pregunta a los miembros del grupo y se va antes de obtener la respuesta por parte de los docentes. El objetivo es que los docentes descubran cuál es el error y puedan corregirlo por sí mismos. Esto genera una tensión en los docentes, pues es habitual que cuando un alumno se equivoca se le hace ver directamente su error. En este caso, "decir" corresponde con indicar directamente los errores y "crecer" con permitir que los estudiantes descubran por sí mismos sus errores.

En los dos episodios siguientes, la tensión surge por las diferencias que los docentes perciben entre su propia disposición al trabajo en grupo y la disposición que en general muestran sus estudiantes a colaborar con otros compañeros. Al igual que el resto de las tensiones de esta sección, son de tipo pedagógico (Nipper et al., 2011) y provienen de la proyección que los docentes hacen del modelo de trabajo en grupo realizado en el Taller RPAula a la realidad de sus aulas (Figura 1).

\section{Episodio 5.}

[M] ¿y qué otras habilidades o conductas, actitudes estamos aprendiendo cuando trabajamos en una dinámica como ésta?

[D1] Bueno, a ser colaborativos. Primero a ser colaborativos entre nosotros. 
[M] Ya, ¿y por qué habría que colaborarle al de al lado?, ¿por qué no resolvía el problema solo? No le hablaba a nadie mejor y levantaba la mano y que me trajeran otro problema.

[D1] Oye, aquí se da inmediatamente en la dinámica y por la forma de ser de cada uno. Pero obviamente cuando tú invitas a un niño, si no lo tiene, así a hacerlo...

[D2] Es innato en algunos, pero hay algunos que no. Algunos son egoístas: que ya aprendieron y no ayudan al resto. Entonces es necesario saber... ¿qué he hecho yo? "iTú sabes!", les digo yo, "Tú sabes. Ayúdame, por favor. Ayúdame, anda y recorre a ver a quién puedes ayudar". Y fíjate que se sienten [...] ¡son mis ayudantes! Y parten. Pero, generalmente aquellos que saben mucho, cuesta sacar el...

\section{Episodio 6.}

[D] Y, también nos pasó que los niños no. Tú los pones de más de dos o más de tres, se arma un revoltijo, en especial si no están acostumbrados.

Los dos episodios que vienen a continuación tienen que ver con la gestión del tiempo y el monitoreo del trabajo de los estudiantes. Sucede uno a continuación del otro, aunque los presentamos separados para mostrar con más claridad cada uno de los focos.

Episodio 7. En esta primera parte, se observa una tensión generada por el tiempo que transcurría desde que un grupo de docentes llamaba al monitor hasta que éste acudía a atenderlos. El episodio hace referencia a que esta situación no debe darse con los alumnos, no deben permitirse los tiempos muertos en el trabajo de los estudiantes.

[D1] Pero yo te hice ver un contratiempo en la mañana. Bueno, en la mañana te lo dije. Digamos que hubo un momento en que, bueno, en la mañana fue como mucho más fácil. Y hubo un momento en que nosotros esperábamos harto tiempo. Esperábamos, esperábamos y tú no dabas a vasto de ir a cada grupo que te llamaba. Entonces había mucho tiempo muerto, donde la persona se empieza a desesperar "ya po, yapo". Pero nosotros, como adultos, claro, esperamos, pero los niños no esperan. 
Episodio 8. La discusión anterior continúa, apareciendo una tensión relacionada con la forma en que debiera monitorearse el trabajo y las dificultades de los estudiantes, sin desatender al resto de los alumnos.

[M] Ahí hay algo clave (refiriéndose al episodio 7) que es cuando... Supongamos que ustedes sean los que resuelvan el problema. ¿Qué es lo que debería pasar? [...] ¿Me debería yo quedar con los grupos? [D1] No po', circular. Es un mediador. Entonces tú vas por cada grupo viendo, guiando, dando las pistas, pero no quedarte ahí, sino movilizarte.

[M] Pero ¿y si yo me movilizo relativamente rápido?

[D2] Pero, pero uno se tiene que quedar cuando hay una dificultad, tal como lo hiciste tú. Te tuviste que quedar un rato para incitar.

[D3] Detenerte ahí. Pero lamentablemente sí tienes que detenerte.

[M] Porque si me detengo pasa lo que dice D1.

[D1] Pero sí te tienes que detener porque aquí pasó lo mismo que pasa en las salas porque hay diferentes [ritmos]. Como dice ella, que hace tiempo no hace matemáticas, [...]en el segundo grupo [...] éramos todas matemáticas medias cuadras y que no nos podía salir el resultado porque buscábamos los factores y los múltiplos y no salíamos de ahí. Entonces, te digo yo, también pasa en los niños. Y pasa en ese niño que no entiende y tú te sientes con el deber de quedarte ahí un rato más, para poderlo guiar, porque ves que está totalmente perdido.

Los últimos cuatro episodios difieren del resto, en el hecho de que son tensiones asociadas a aspectos del taller que los docentes parecen sentir que no funcionarán en sus clases. Estarían en el grupo de lo que Nipper et al. (2011) denominan "tensiones pedagógicas aplicativas". Durante el desarrollo de la sesión, los docentes identifican aspectos de la metodología del taller que consideran adecuados para ellos por ser adultos, pero que consideran inadecuados o complejos de implementar con sus estudiantes, como dejarlos solos un tiempo, sin atender a sus Ilamadas, o hacerlos trabajar en grupo. En este sentido, podrían convertirse en motivos para la resistencia al cambio, al menos hasta que los docentes pongan en práctica el modelo con sus estudiantes y observen cómo funciona (Guskey, 2002). 
La resolución de problemas como estrategia de desarrollo profesional docente:

tensiones que se generan en el profesor

Cristóbal Rojas, Josefa Perdomo-Díaz y Patricio luis Felmer

\section{Tensiones en la relación Docente-Sistema}

Por último, mostraremos dos episodios en los que se observa una tensión que no puede asociarse a ninguna de las relaciones establecidas a priori en el modelo de relaciones del Taller RPAula (Figura 1), ya que se da en la relación del docente con el sistema. La tensión se produce por la discrepancia que puede existir entre la visión de los compañeros y superiores, sobre el orden y el ruido que puede permitirse en una clase, y lo que generan dinámicas de trabajo como la usada en el Taller RPAula.

\section{Episodio 9.}

[D] Hay un temor también y eso se debe, bajo mi punto de vista, a varias razones. Primero, cuando tú tienes un gran número de alumnos en la sala y tú sabes que de repente se portan mal, tienes miedo al desorden, tienes miedo a [...] que te digan... A mí, como profesor [...] o sea, nosotros, como estamos tan sujetos a la crítica, no ven lo positivo que estamos haciendo. Porque los niños pueden estar en el suelo, haciendo un trabajo, pero se pasa un colega que tiene otra mirada, y ve que tú estás en un desorden y los cabros no están haciendo nada. Entonces, nosotros nos hemos ido quedando en un espacio más reducido y nos movemos lo menos posible. Entonces, los chiquillos también. Entonces, vieras tú, están todos sentados ahí con su lápiz y vamos haciendo los ejercicios y que nadie se mueva de ahí.

\section{Episodio 10.}

[D1] Eso es lo que yo le decía recién. Que yo me pongo [una] estructura [...] Bueno ya, como decían los demás. Que entra alguien de la inspección y te ve [...] Y están trabajando todos ahí estructuraditos, están trabajando, están en grupos y están desordenados, pero están en grupo. [D2] Pero, se desordenan pero trabajan. Porque se desordenan para el trabajo. A nosotros nos pasa lo mismo. Si a nosotros acá nos hubieran sentado así en fila, todos así y vamos a trabajar, más callados quizás. Pero, en grupo, trabajamos todos igual, independiente del grupo que te vaya a tocar, trabajamos igual. Y también nos pasó.

$[\mathrm{M}]$ ¿Y qué ventajas tiene?

[D3] Y, también nos pasó que los niños no. Tú los pones de más de dos o más de tres, se arma un revoltijo, en especial si no están acostumbrados. 
Estas tensiones podrían asociarse a lo que Rouleau y Liljedhal (2017) denominan Iniciativa versus Barreras Sistémicas. Sin embargo, las manifestaciones de los docentes en este taller acusan esa tensión a la incomodidad o el rechazo que la innovación pueda provocar en sus superiores, directivos, inspectores, o en sus compañeros.

\section{Discusión final}

El diseño del Taller RPAula incluye varios de los elementos que la literatura asocia a los PDP de calidad (Borko, 2004; Garet et al., 2001; Marrongelle et al., 2013): busca que los docentes profundicen en sus conocimientos acerca de la resolución de problemas y reflexionen sobre los procesos de aprendizaje de los estudiantes, modificando algunos elementos de sus prácticas. Sin embargo, esto no es garantía de que se produzcan cambios en los docentes; para ello es necesario que los participantes reconozcan las brechas existentes entre sus conocimientos, creencias y prácticas y lo que se presenta en el PDP (Ávalos, 2011). El principal objetivo de la primera sesión del taller es precisamente hacer explícitas algunas de esas fisuras, activar los mecanismos para que los participantes tomen conciencia de los cambios que tendrían que realizar para implementar en su aula el modelo de trabajo en resolución de problemas, propuesto en el taller.

Los resultados obtenidos son coherentes con los objetivos de la primera sesión del Taller RPAula. En esta sesión se sitúa a los profesores en el papel de resolutores de problemas, con un doble propósito: que tomen conciencia de su propia habilidad para resolver problemas matemáticos y que analicen la dinámica de trabajo.

En el caso estudiado, entre los participantes afloraron tanto tensiones matemáticas como pedagógicas (Nipper et al., 2011). Las matemáticas, relacionadas con los conocimientos y habilidades para la resolución de problemas que los docentes comprobaron que tenían (episodios 1 y 2). Para enseñar resolución de problemas, los profesores necesitan disponer de un conocimiento propio como resolutores de problemas, además de conocimientos específicos sobre su enseñanza (Chapman, 2015); reconocer ciertas limitaciones en los propios conocimientos de la disciplina sienta unas bases positivas para iniciar el camino hacia el cambio. Las actividades planteadas, el trabajo en grupo y la discusión final, fueron 
La resolución de problemas como estrategia de desarrollo profesional docente:

los elementos del Taller RPAula que llevaron a los participantes a reflexionar sobre sus conocimientos y habilidades matemáticas.

Las tensiones pedagógicas manifestadas por los docentes tuvieron que ver con la respuesta al error o a las dudas de los estudiantes (episodios 3 y 4), relacionadas con la dicotomía entre indicar a los alumnos cómo se hacen las cosas o no, ofreciéndoles oportunidades para que aprendan por sí mismos, lo que Berry (2008) denominó "Decir versus Crecer"; el trabajo en grupo (episodios 5 y 6) y la gestión del tiempo y el monitoreo a los alumnos (episodios 7 y 8). Estas tensiones afloraron a raíz del tipo de interacción que el modelo del Taller RPAula muestra, tanto entre los profesores como con el monitor, y que los docentes proyectan a la realidad de su aula (Nipper \& Sztajn, 2008).

Los profesores también hicieron referencia a su preocupación por cómo algunos miembros del sistema educativo percibirían el modelo de trabajo propuesto, lo que muestra tensiones en la relación con el sistema y las barreras que los docentes sienten que les pone su contexto (Rouleau y Liljedhal, 2017), lo que justifica la necesidad de profundizar en este tipo de investigación, realizada desde una perspectiva situada (Borko, 2004).

El modelo de relaciones asociado al diseño del Taller RPAula (Figura 1) resultó de gran utilidad en esta investigación y constituye una herramienta para el desarrollo del propio taller. Algunas de las tensiones identificadas en este trabajo son abordadas a lo largo de las otras siete sesiones del taller (Perdomo-Díaz \& Felmer, 2017), otras aportan información para el diseño de propuestas que complementen a ésta, como por ejemplo, el diseño de talleres con foco en el conocimiento matemático para la enseñanza de la resolución de problemas (Chapman, 2015).

\section{Agradecimientos}

J.P-D ha sido parcialmente financiada por el proyecto EDU201784276-R del Plan Nacional de I+D+I del Programa Estatal de Investigación, Desarrollo e Innovación orientada a los retos de la sociedad del Ministerio de Economía y Competitividad. P.F. ha sido parcialmente financiado por PIA-CONICYT Financiamiento Basal para Centros de Excelencia, Proyecto FB0003. 
La resolución de problemas como estrategia de desarrollo profesional docente: tensiones que se generan en el profesor Cristóbal Rojas, Josefa Perdomo-Díaz y Patricio luis Felmer

\section{Referencias}

Ávalos, B. (2011). Teacher professional development in Teaching and Teacher Education over ten years. Teaching and Teacher Education, 27, 10-20.

Berry, A. (2008). Tensions in Teaching about Teaching: Understanding Practice as a Teacher Educator. Dordrecht, The Netherlands: Springer.

Borko, H. (2004). Professional Development and Teacher Learning: Mapping the Terrain. Educational Researcher, 33 (8), 3-15.

Brown, R. E. (2010). Tensions faced by mathematics professional developers. The Mathematics Educator, 20 (1), 3-7.

Chapman, O. (2015). Mathematics teachers' knowledge for teaching problem solving. LUMAT, 3(1), 19-36.

Felmer, P., Pehkonen, E. \& Kilpatrick, J. (Eds.) (2016). Posing and Solving Mathematical Problems. Advances and New Perspectives. Switzerland: Springer.

Garet, M.S., Porter, A.C., Desimone, L., Birman, B.F., \& Suk Yoon, K. (2001). What Makes Professional Development Effective? Results from a National Sample of Teachers. American Educational Research Journal, 38, 915-945. DOI: 10.3102/00028312038004915

Guskey, T.R. (2002). Professional Development and Teacher Change. Teachers and Teaching: theory and practice, 8 (3/4), 381-391. DOI: 10.1080/135406002100000512

Lampert, M. (1985). How do teachers manage to teach? Perspectives on problems in practice. Harvard Educational Review, 55(2), 178-195.

Liljedahl, P., Santos-Trigo, M., Malaspina, U. y Bruder, R. (Eds.). (2016). Problem Solving and Mathematics Education. Switzerland: Springer. DOI: 10.1007/978-3-319-40730-2

Marrongelle, K., Sztajn, P., \& Smith, M. (2013). Scaling Up Professional Development in an Era of Common State Standards. Journal of Teacher Education, 64, 202-211- DOI: 10.1177/0022487112473838

Nipper, K., Ricks, T., Kilpatrick, J., Mayhew, L., Thomas, S., Kwon, N. Y., Klerlein, J. T., Hembree, D. (2011). Teacher tensions: expectations in a professional development institute. Journal of Mathematics Teacher Education, 14 (5), 375-392.

Nipper, K., y Sztajn, P. (2008). Expanding the instructional triangle: Conceptualizing mathematics teacher development. Journal of Mathematics Teacher Education, 11 (4), 333341.

Perdomo-Díaz, J. \& Felmer, P. (2017). El taller RPaula: activando la resolución de problemas en las aulas. Profesorado. Revista de currículum y formación del profesorado, 21 (2), 425-444.

Rouleau, A. (2017). Tensions in the role of mathematics coaches. En T. Dooley y G. Gueudet (Eds.), Proceedings of the 10th Congress of European Research in Mathematics Education (CERME) (pp. 2988-2995). Dublin, Ireland: DCU Institute of Education and ERME.

Rouleau, A., y Liljedahl, P. (2017). Teacher Tensions: The Case of Naomi. En C. Andrà, D. Brunetto, E. Levenson y P. Liljedahl (Eds.), Teaching and Learning in Maths Classrooms. Research in Matematics Education (pp. 155-162). Cham: Springer. DOI: https://doi. org/10.1007/978-3-319-49232-2_15 
La resolución de problemas como estrategia de desarrollo profesional docente: tensiones que se generan en el profesor

Cristóbal Rojas, Josefa Perdomo-Díaz y Patricio luis Felmer

Tan, A. L., Chang, C. H., y Teng, P. (2015). Tensions and dilemmas in teacher professional development. Procedia-Social and Behavioral Sciences, 174, 1583-1591. 